\title{
Quantitation of glucose uptake in tumors by dynamic FDG-PET has less glucose bias and lower variability when adjusted for partial saturation of glucose transport
}

Simon-Peter Williams ${ }^{1 *}$, Judith E Flores-Mercado ${ }^{1}$, Ruediger E Port ${ }^{2}$ and Thomas Bengtsson ${ }^{3^{*}}$

\begin{abstract}
Background: A retrospective analysis of estimates of tumor glucose uptake from 1,192 dynamic 2-deoxy-2-( $\left.{ }^{18} \mathrm{~F}\right)$ fluoro-D-glucose-positron-emission tomography [FDG-PET] scans showed strong correlations between blood glucose and both the uptake rate constant $\left[K_{i}\right]$ and the metabolic rate of glucose [MRGluc], hindering the interpretation of PET scans acquired under conditions of altered blood glucose. We sought a method to reduce this glucose bias without increasing the between-subject or test-retest variability and did this by considering that tissue glucose transport is a saturable yet unsaturated process best described as a nonlinear function of glucose levels.

Methods: Patlak-Gjedde analysis was used to compute $K_{\mathrm{i}}$ from 30-min dynamic PET scans in tumor-bearing mice. MRGluc was calculated by factoring in the blood glucose level and a lumped constant equal to unity. Alternatively, we assumed that glucose consumption is saturable according to Michaelis-Menten kinetics and estimated a

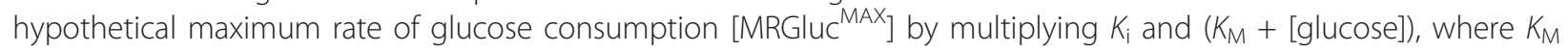
is a half-saturation Michaelis constant for glucose uptake. Results were computed for 112 separate studies of 8 to 12 scans each; test-retest statistics were measured in a suitable subset of 201 mice.

Results: A $K_{\mathrm{M}}$ value of $130 \mathrm{mg} / \mathrm{dL}$ was determined from the data based on minimizing the average correlation between blood glucose and the uptake metric. Using MRGluc ${ }^{\mathrm{MAX}}$ resulted in the following benefits compared to using MRGluc: (1) the median correlation with blood glucose was practically zero, and yet (2) the test-retest coefficient of variation [COV] was reduced by $13.4 \%$, and (3) the between-animal COVs were reduced by $15.5 \%$. In statistically equivalent terms, achieving the same reduction in between-animal COV while using the traditional MRGluc would require a $40 \%$ increase in sample size.

Conclusions: MRGluc appeared to overcorrect tumor FDG data for changing glucose levels. Applying partial saturation correction using MRGluc ${ }^{\mathrm{MAX}}$ offered reduced bias, reduced variability, and potentially increased statistical power. We recommend further investigation of $M R G l u C^{M A X}$ in quantitative studies of tumor FDG uptake.
\end{abstract}

Keywords: variability, glucose correction, MRGluc ${ }^{\mathrm{MAX}}$, blood glucose, partial saturation correction, dynamic FDG-PET

\footnotetext{
* Correspondence: williams.simon@gene.com; bengtsson.thomas@gene.com

1 Department of Biomedical Imaging, Genentech, Inc., South San Francisco,

CA, 94080, USA

${ }^{3}$ Department of Biostatistics, Genentech, Inc., South San Francisco, CA, 94080,

USA

Full list of author information is available at the end of the article
} 


\section{Background}

We considered 2-deoxy-2- $\left({ }^{18} \mathrm{~F}\right)$ fluoro-D-glucose-positron-emission tomography [FDG-PET] as a pharmacodynamic marker of antitumor activity during treatments that alter systemic blood glucose levels, for example the Akt inhibitors [1], and sought a metric of tumor glucose uptake that had minimal glucose bias. Inverse correlations of blood glucose with tumor FDG uptake have been demonstrated in multiple settings (see Figures 1 and 2, [2-6]), and this effect was to be expected based on the biochemistry of glucose (and tracer) transport and trapping [7].

We undertook a large series of tumor imaging studies in mice using the metabolic rate of glucose [MRGluc] from Patlak analysis as our preferred estimate of the tumor glucose uptake rate, expecting it to be relatively unbiased with respect to blood glucose. When we undertook a retrospective review of 1,192 such scans performed in study groups of 8 to 12 mice, we observed that our MRGluc data were, in fact, strongly correlated with blood glucose even though individual studies were often underpowered to convincingly show this (see Figure $2 \mathrm{~B}$ ).

We presumed that this correlation caused additional variability in the uptake measurements. Even in the absence of any active treatment, blood glucose levels were not entirely constant in our studies (see Figure 3), so we sought to apply a rational glucose correction to the MRGluc data, noting that the bias reduction benefit

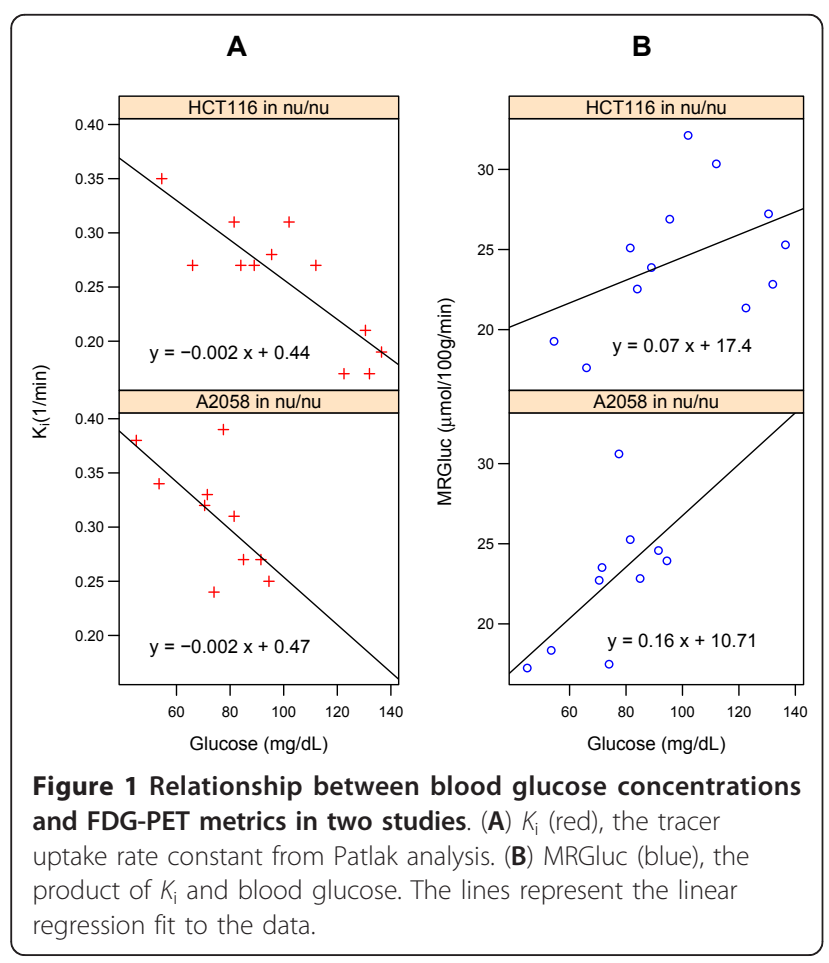

must outweigh the cost of the statistical noise introduced by the blood glucose measurements [8].

The original formulations of quantitative glucose uptake measurements using radioactive uptake assays were described comprehensively 35 years ago in the seminal work of Sokoloff et al. [7]. The importance of glucose transport processes based on saturable Michaelis-Menten kinetics has been demonstrated in biochemical studies of glucose transporter 1 [GLUT-1], the dominant glucose transporter in tumors (and erythrocytes and the blood-brain barrier), which have shown that glucose transport into cells can be characterized as a saturable process with a half-maximal-rate Michaelis constant $\left[K_{\mathrm{M}}\right]$ of approximately $40 \mathrm{mg} / \mathrm{dL}$ [9]; it is the transport step that dominates the overall uptake and trapping rate in many situations $[10,11]$. Studies in intact animals suggested that the apparent half-saturation constant, $K_{\mathrm{M}}$, for the GLUT-1-dominated blood-tobrain tissue transport was approximately 5 [12] to 7.3 $\mathrm{mM}$ [13], equivalent to 100 to $130 \mathrm{mg} / \mathrm{dL}$.

We reasoned that tissue glucose levels are often neither far below $K_{\mathrm{M}}$ (where the glucose transport rate would be approximately proportional to blood glucose level) nor far above $K_{\mathrm{M}}$ (where the glucose transport rate would be saturated and independent of blood glucose level). Consequently, tissue glucose uptake rates are likely to show an intermediate, nonlinear dependence on blood glucose levels.

We tested this hypothesis using a form of the MRGluc calculation that employs the Michaelis-Menten relationship to compute the hypothetical maximal uptake rate [MRGluc ${ }^{\mathrm{MAX}}$ ] based on an empirical half-saturation $K_{\mathrm{M}}$ of $130 \mathrm{mg} / \mathrm{dL}$ (see 'Results' section). This approach should reflect the relatively constant glucose uptake capacity of the tissue rather than the instantaneous uptake rate, more or less independent of variations in blood glucose. We refer to this glucose correction method as partial saturation correction.

In this paper, we review 112 separate tumor studies of 8 to 12 dynamic FDG scans each, all analyzed with the Patlak-Gjedde simplified tracer kinetic modeling methods [14-17] yielding the uptake rate constant $\left[K_{\mathrm{i}}\right]$ (per second) and the MRGluc (in micromoles per minute per $100 \mathrm{~cm}^{3}$ ). We compared the glucose bias, test-retest, and between-animal variability of $K_{\mathrm{i}}$, MRGluc, and MRGluc $^{\text {MAX }}$.

\section{Materials and methods Data enrollment}

The data retrospectively analyzed here came from 11 different xenograft models of human cancers that we have employed in recent projects. Each model is a unique combination of a mouse strain and a tumor line. Included were (1) scans from mice studied at baseline 


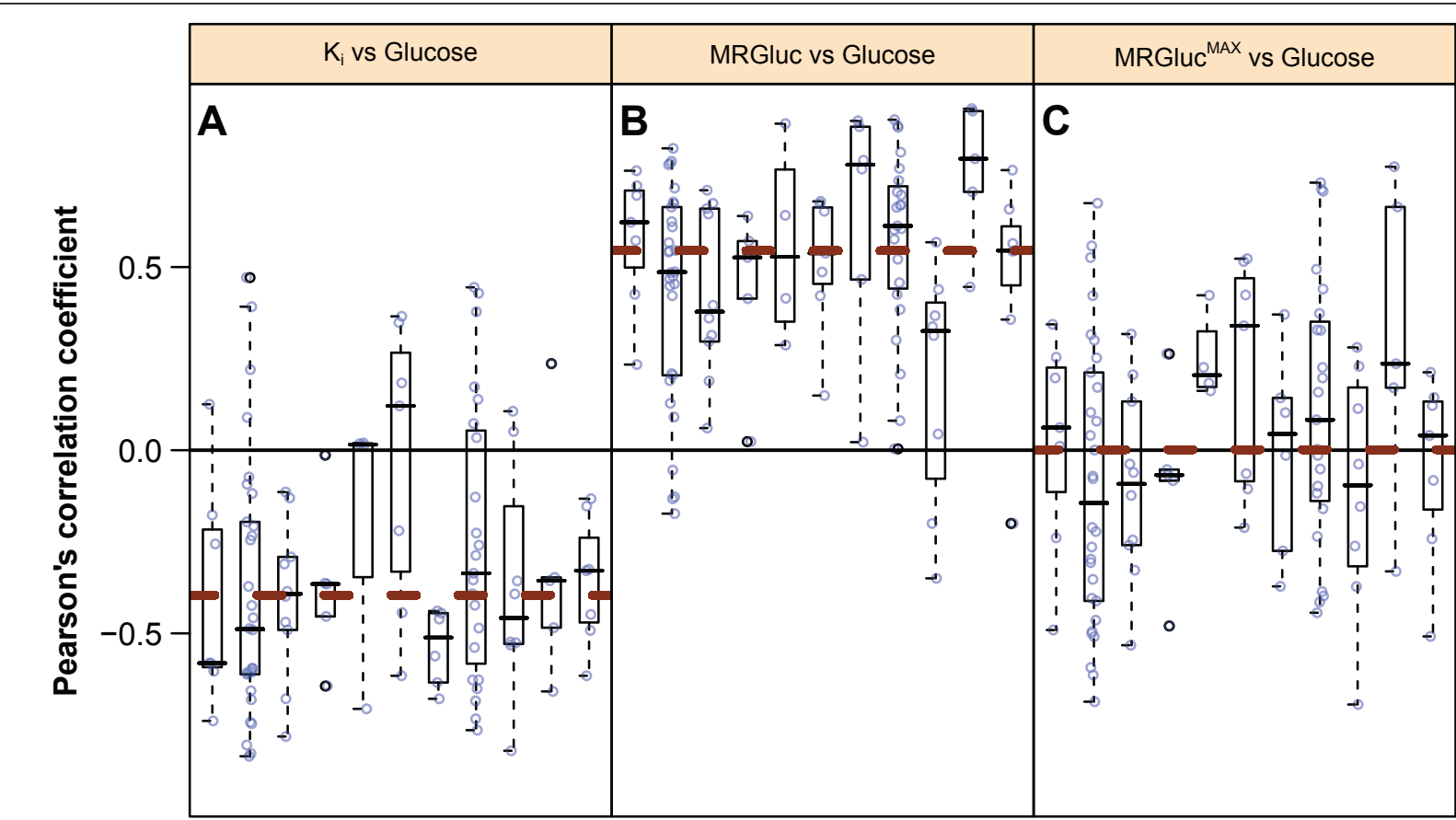

A B CDEFGH I JKA BCDEFGH I JKABCDEFGH I J K

\section{Animal model}

Figure 2 Pearson's correlation coefficients between blood glucose and $K_{\mathrm{i}}$ (A), MRGluc (B), and MRGluc ${ }^{\text {MAX }}$ (C). The $y$-axis represents Pearson's correlation value obtained in 112 studies (1,192 observations-see 'Materials and methods' section). The $x$-axis indicates the 11 tumor models (see Table 1). Open circles represent correlation coefficients of individual studies. Box plots show the 25th, 50th, and 75th percentiles of correlation coefficient distributions for every animal model. The thick brown dashed lines show the median for all data.

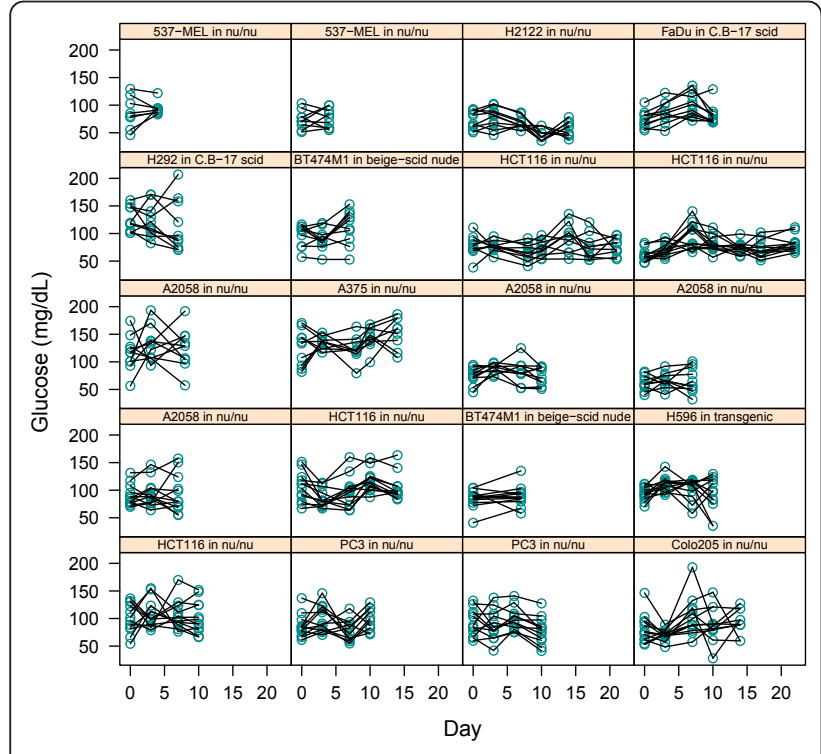

Figure 3 Individual mouse blood glucose levels (y-axis) on multiple scanning days ( $\boldsymbol{x}$-axis). Mean scan-time glucose was calculated as the average of the pre-scan and post-scan measurements. Each box corresponds to a different cohort of mice as noted. prior to any treatment and (2) any subsequent scans from mice enrolled in control groups not receiving any drug substance. Table 1 describes the 585 mice and 1,192 scans that were included. The mice were studied as cohorts of 8 to 12 individuals. Each member of a cohort had the same gender, age, strain, and tumor type, and they were raised and inoculated at the same time. The average tumor volume in a cohort was 250 to $400 \mathrm{~mm}^{3}$ at the beginning of an imaging experiment. A study is defined here as the imaging of one cohort at one timepoint.

\section{Imaging}

All studies were conducted with the approval of Genentech's AALAC-accredited institutional animal care and use committee. Briefly, animals were fasted overnight with free access to water prior to PET imaging. Sevoflurane in air [18] was used to induce and maintain anesthesia sufficient to restrain the animals while they were scanned prone on the bed of an Inveon MM scanner (Siemens Preclinical Solutions, Knoxville, TN, USA). PET scans lasted $30 \mathrm{~min}$. X-ray CT scans provided attenuation correction. List mode data were typically reconstructed into images with $128 \times 128$ in-plane 
Table 1 Data listed by mouse strains and tumor types

\begin{tabular}{|c|c|c|c|c|}
\hline Model & Tumor cell line/mouse strain & Tissue of origin & Number of mice & Number of scans \\
\hline A & BT474MI in beige-scid nude & Breast & 44 & 76 \\
\hline B & HCT116 in athymic nude & Colon & 124 & 339 \\
\hline C & PC3 in athymic nude & Prostate & 48 & 116 \\
\hline D & FaDu in C.B.-17 scid & Pharynx & 20 & 50 \\
\hline$E$ & H292 in C.B.-17 scid & Lung & 20 & 40 \\
\hline $\mathrm{F}$ & H596 in huHGF transgenics & Lung & 46 & 81 \\
\hline G & 537-MEL in athymic nude & Skin & 35 & 52 \\
\hline $\mathrm{H}$ & A2058 in athymic nude & Skin & 144 & 236 \\
\hline । & A375 in athymic nude & Skin & 40 & 75 \\
\hline J & Colo205 in athymic nude & Colon & 24 & 58 \\
\hline \multirow[t]{2}{*}{ K } & H2122 in athymic nude & Lung & 40 & 69 \\
\hline & Total & & 585 & 1,192 \\
\hline
\end{tabular}

voxels of $0.4 \times 0.4 \mathrm{~mm}$ and $0.8 \mathrm{~mm}$ through-plane voxel thickness using vendor-provided iterative OP-MAP implementation with the beta hyperparameter set to 0.05 [19]. The resolution (approximately $1.5 \mathrm{~mm}$ ), sensitivity, and other performance characteristics of this scanner have been described previously [20]. Body temperature was maintained at $37^{\circ} \mathrm{C}$ by warm air flows under feedback control. When animals were re-scanned on the second or subsequent days, they were imaged on the same scanner and at the same time of the day as for their first scan. The mice received an FDG tracer dose of approximately $200 \mu \mathrm{Ci}$ by infusion through a tail vein catheter.

\section{Blood glucose measurements}

At every scan, blood glucose measurements were taken twice: once approximately $5 \mathrm{~min}$ before and once shortly after the scan approximately $35 \mathrm{~min}$ later. The glucose value used in calculations is the mean of the pre- and post-scan measurements. Data were collected with the commercially available Contour glucometer (Bayer Healthcare, Tarrytown, NY, USA). Test-retest reproducibility measurements according to Equation 5 were conducted using this instrument in 20 mice and showed a coefficient of variation of $3.7 \%$.

\section{Image analysis}

Regions of interest [ROIs] were drawn using the image analysis software IRW from Siemens. For any given tumor model, all scans for all animals were analyzed by a single observer following a standard procedure: Tumor ROIs were defined as voxels exceeding a threshold percentage of the maximal tumor signal measured in the last $10 \mathrm{~min}$ of the scan; this excluded necrotic or otherwise hypointense regions from the analysis. Mean signal values from the ROIs were used for analysis. Image-derived signal from an ROI in the liver was used as an input function reference region in the Patlak analysis, a technique described in mice by Green et al. [21]. This method is well suited to high-resolution whole-body scans that minimize partial volume artifacts $[20,22]$, such as those used here.

\section{Time-activity curves and Patlak plots}

The Patlak and subsequent statistical analyses were performed with the statistical programming language $\mathrm{R}$ [23]. For each tumor model described in Table 1, examples of the time-activity curves and the resultant Patlak plots are presented in Additional file 1 to $11 . K_{\mathrm{i}}$ was measured from Patlak plots of dynamic FDG-PET data $[15,16,24]$. The linear portion of the plot (beginning approximately $5 \mathrm{~min}$ into the time-activity curves) was used for fitting and visually verified: the correlation coefficient $r^{2}$ in each case was at least 0.99 .

\section{Kinetic modeling and partial saturation correction}

MRGluc was estimated as $K_{\mathrm{i}} \times$ [glucose] $\times$ LC, where LC is the lumped kinetic constant (set to unity) and [glucose] is the blood glucose measurement. Some literature denotes this form of MRGluc where $L C=1$ as 'MRFDG' [25,26]; we will use 'MRGluc' for its semantic emphasis on glucose (rather than FDG or glutamate) uptake. Although the LC scales the absolute value of the $K_{\mathrm{i}}$ data, it is important to note that the choice of LC has no bearing on the subsequent analysis of glucose correlation, between-animal coefficient of variation [COV], or test-retest reproducibility.

MRGluc and its basic dependence on blood glucose levels were modeled according to Equation 1, a form of the Michaelis-Menten relationship [27,28]:

$$
\text { MRGluc }=\frac{\text { MRGluc } \left.^{\mathrm{MAX}} \text { [glucose }\right]}{\left.K_{\mathrm{M}}+\text { [glucose }\right]} .
$$

MRGluc $^{\mathrm{MAX}}$ is the hypothetical maximal value of glucose uptake rate, approached asymptotically as the 
glucose concentration increases to saturating levels. If it were physically possible, the glucose uptake rate measured along the horizontal asymptote would be expected to show zero correlation with the glucose concentration. The curvature parameter $K_{\mathrm{M}}$ is the Michaelis constant that represents the blood glucose concentration at which the glucose uptake rate is half the maximal (glucose-saturated) rate.

To see if our data plausibly followed the MichaelisMenten model, we transformed the measurements into the linear double-reciprocal form of Equation 2 and generated the corresponding Lineweaver-Burk plots (some examples are shown in Figure 4):

$$
\frac{1}{\text { MRGluc }}=\frac{K_{\mathrm{M}}}{\text { MRGluc }^{\mathrm{MAX}}} \frac{1}{\text { [glucose] }}+\frac{1}{\text { MRGluc }^{\mathrm{MAX}}} \text {. }
$$

\section{Computation of MRGluc ${ }^{\text {MAX }}$}

We divide both sides of Equation 1 by [glucose] and see that

$$
K_{\mathrm{i}}=\operatorname{MRGluc}^{\mathrm{MAX}}\left(\frac{1}{\left.K_{\mathrm{M}}+\text { [glucose }\right]}\right) .
$$

A further rearrangement allows the computation of MRGluc $^{\mathrm{MAX}}$ for each individual animal:

$$
\text { MRGluc } \left.^{\mathrm{MAX}}=K_{\mathrm{i}}\left(K_{\mathrm{M}}+\text { [glucose }\right]\right) .
$$

All the data presented in this paper were computed using Equation 4, and group mean data were calculated by sample averaging the results for individual animals within a given study.

\section{Estimation of $K_{\mathrm{M}}$ by minimizing the correlation between blood glucose and MRGluc ${ }^{\text {MAX }}$}

We computed estimates of MRGluc ${ }^{\mathrm{MAX}}$ with a range of $K_{\mathrm{M}}$ values from 40 to $200 \mathrm{mM}$ and selected the $K_{\mathrm{M}}$ that gave the smallest nonnegative value of the median

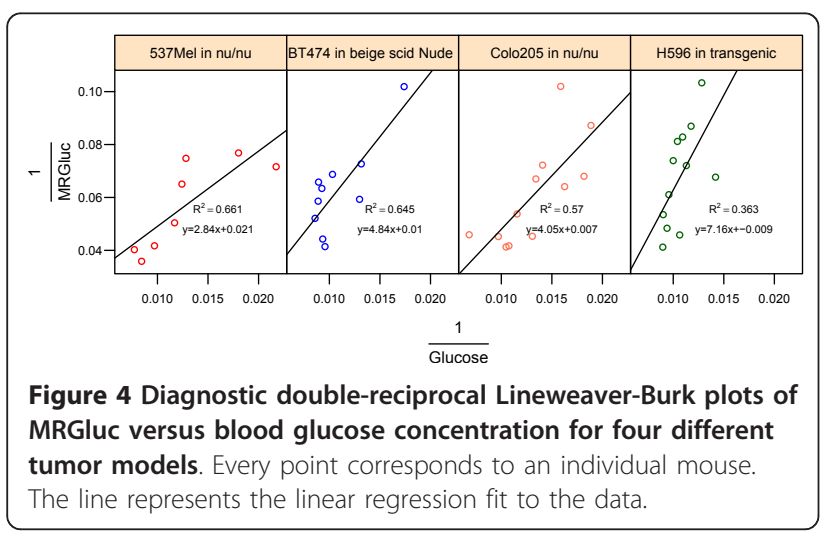

Pearson's correlation coefficient between MRGluc ${ }^{\text {MAX }}$ and [glucose] across all 112 studies. As an exploratory analysis, we also separately estimated $K_{\mathrm{M}}$ for each of the 11 tumor models.

\section{Variability and reproducibility}

Between-animal variability was measured as the COV, calculated as standard error of the estimate divided by the estimate, and expressed as a percentage. Test-retest reproducibility statistics were calculated for 19 studies with the 201 mice that were scanned at day 0 and again at day 3. This was the most common test-retest interval in our data. The COVs were calculated using Equation 5, as described by Weber et al. [29]:

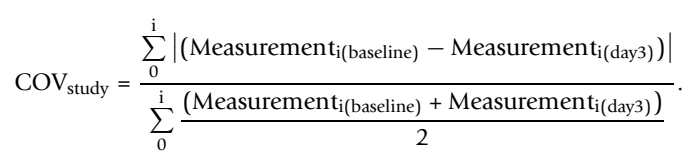

\section{Results}

Both $K_{\mathrm{i}}$ and MRGluc are correlated with blood glucose levels

In some studies, correlations between blood glucose levels and the FDG-PET estimates of glucose uptake rate were readily apparent. Two of these are illustrated in Figure 1: panel A for $K_{\mathrm{i}}$ and panel B for MRGluc. As expected, many individual cohorts of 8 to 12 mice were statistically underpowered to show such a relationship.

More important, and remarkable, was the consistent presence and strength of this relationship between blood glucose and tissue uptake rates when seen in the metaanalysis of our large sample of studies. Figure 2 illustrates this using a box plot of Pearson's correlation coefficients between blood glucose and (A) $K_{\mathrm{i}}$, and (B) MRGluc for all 1,192 scans from the 112 studies. The data are grouped into one box for each of the 11 tumor models, with the median for each box shown as a horizontal line. Data for each tumor model comprised 4 to 30 studies; the open circles within a box show individual studies for full disclosure.

Blood glucose levels were negatively correlated with $K_{\mathrm{i}}$ in 90 studies (Figure 2A). The median correlation coefficient (dashed line) was -0.4. With MRGluc as the metric of tumor glucose uptake rate, 104 studies now showed a positive correlation with a median correlation coefficient of 0.55 (Figure 2B), indicating that factoring in the glucose did not eliminate the bias, but rather changed it from negative to positive.

In this meta-analysis of 112 studies, it is possible to compute for each tumor model confidence interval around the correlation coefficients reported in Figure 2. The statistical methodology and results are presented for the interested reader in Additional file 2. 


\section{Lineweaver-Burk plots}

A preliminary analysis of our data simply looked for positive correlations between MRGluc and blood glucose levels in the double-reciprocal Lineweaver-Burk plots that are characteristic of a Michaelis-Menten relationship [27,28]. Thirteen of the first 20 tumor studies we examined had some correlation, judging by eye, encouraging further consideration of the MichaelisMenten model in our data. It was also apparent that the data were inherently noisy such that individual studies were perhaps underpowered to demonstrate a relationship. No quantitative inferences were drawn from these analyses, however. Four such studies are shown in Figure 4.

\section{When $K_{\mathrm{M}}=130 \mathrm{mg} / \mathrm{dL}$, MRGluc ${ }^{\mathrm{MAX}}$ shows zero glucose bias, on average}

Figure $2 \mathrm{C}$ shows the correlation between MRGluc ${ }^{\mathrm{MAX}}$ and blood glucose when $K_{\mathrm{M}}=130 \mathrm{mg} / \mathrm{dL}$. At this value of $K_{\mathrm{M}}$, the median correlation coefficient for all 112 studies was practically equal to zero, $<0.0004$ (dashed line in Figure 2C). Of the 112 studies, 55 showed a positive correlation, 55 showed a negative correlation, and 2 had practically zero correlation $(<0.003)$. Increasing values of $K_{\mathrm{M}}$ beyond $130 \mathrm{mg} / \mathrm{dL}$ resulted in progressively more negative median correlation coefficients.

\section{Individual blood glucose often varies between scans}

Blood glucose levels recorded at scan time for individual mice on multiple measurement days are presented in Figure 3. Each box contains a different cohort of mice studied on multiple days. Differences in group means and fluctuations over time are apparent despite consistency of handling.

\section{Between-animal variability}

From Figure 5A, we observe that there was typically a reduction in the COV of MRGluc ${ }^{\mathrm{MAX}}$ with respect to the COV of the same scans quantified using MRGluc. Most of the points lie below the identity line; 87 of the 112 studies analyzed showed some improvement. The average reduction in COV was measured as $15.5 \%$ from the value of the fitted regression line slope of 0.845 shown as the dashed line in Figure 5A.

Our hypothesis was that we could reduce variability by extrapolating the tumor glucose uptake rate measurement to a hypothetical asymptote where glucose is under saturating conditions, and our data seem to support this. Mathematically, the improvement in $\mathrm{COV}$ appears to come from the fact that MRGluc ${ }^{\mathrm{MAX}}$ is greater than MRGluc by definition. Specifically, because $K_{\mathrm{M}}=130 \mathrm{mg} / \mathrm{dL}$ and the glucose measurements are near $100 \mathrm{mg} / \mathrm{dL}$, we observe that, on the average, MRGluc $^{\text {MAX }}$ values approximately double those of MRGluc. The standard error in MRGluc ${ }^{\mathrm{MAX}}$ is also greater than that in MRGluc, but proportionately less so, and so we get an overall reduction in COV of $15.5 \%$.

\section{Power estimation and sample size calculation}

An overall reduction in COV of $15.5 \%$ could translate (statistically equivalently) into either a need for fewer subjects per study or into the ability to detect smaller
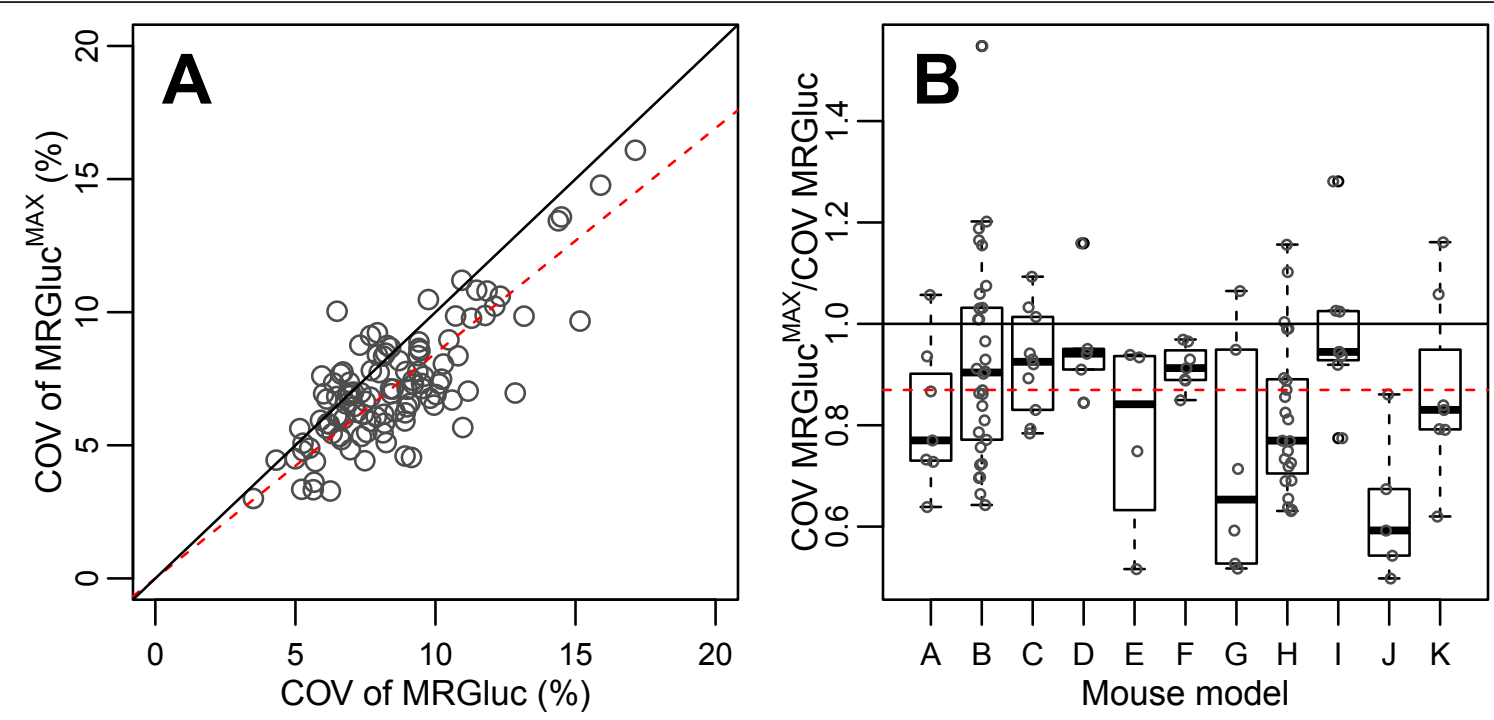

Figure 5 COVs in 112 FDG studies of 8 to 12 scans. Each COV was calculated with (MRGluc ${ }^{\text {MAX) }}$ ) or without (MRGluc) partial saturation correction. For MRGluc ${ }^{M A X}$, a $K_{M}$ of $130 \mathrm{mg} / \mathrm{dL}$ was used. Each open circle represents the COV calculated for one study. (A) Scatter plot of all 112 observations. The solid line represents the identity line. The red dashed line below the identity line represents the linear regression fit to the data. (B) Box plots showing the COV ratios grouped according to the 11 mouse models employed. The red dashed horizontal line represents the median of all values. 
effect sizes. Generally speaking, the standard error of sample means (and of maximum likelihood estimators in general) is inversely proportional to the square root of the sample size: this implies that to achieve the same between-animal COV when using MRGluc would require an increased sample size of $40 \%$ (i.e., $100 \times 1$ / $\left.(1-0.155)^{2}\right)$ compared when using MRGluc ${ }^{\mathrm{MAX}}$.

\section{Test-retest reproducibility}

For the 19 studies examined, the median test-retest reproducibility COV results were $22.0 \%$ for $K_{\mathrm{i}}, 23.1 \%$ for MRGluc, and $20.0 \%$ for MRGluc ${ }^{\text {MAX }}$. Figure 6 illustrates the distribution of $\mathrm{COV}$ values for each of the three PET metrics.

\section{Sensitivity analysis for the between-animal COV as a function of $K_{\mathrm{M}}$}

Varying the value of $K_{\mathrm{M}}$ in the range of 40 to $200 \mathrm{mg} /$ $\mathrm{dL}$ did not change the nature of the results: MRGlucMAX gave lower between-animal variability than MRGluc. The reduction in the average $\mathrm{COV} / K_{\mathrm{M}}$ correspondence was $10 \%(40 \mathrm{mg} / \mathrm{dL}), 15 \%(100 \mathrm{mg} / \mathrm{dL})$, $15.5 \%(130 \mathrm{mg} / \mathrm{dL})$, and $16 \%(200 \mathrm{mg} / \mathrm{dL})$.

\section{Discussion}

\section{Correlations between blood glucose levels and MRGluc}

Figure 2B shows that, in our setting with anesthetized mice, there is undoubtedly a strong and persistent positive correlation between blood glucose and MRGluc

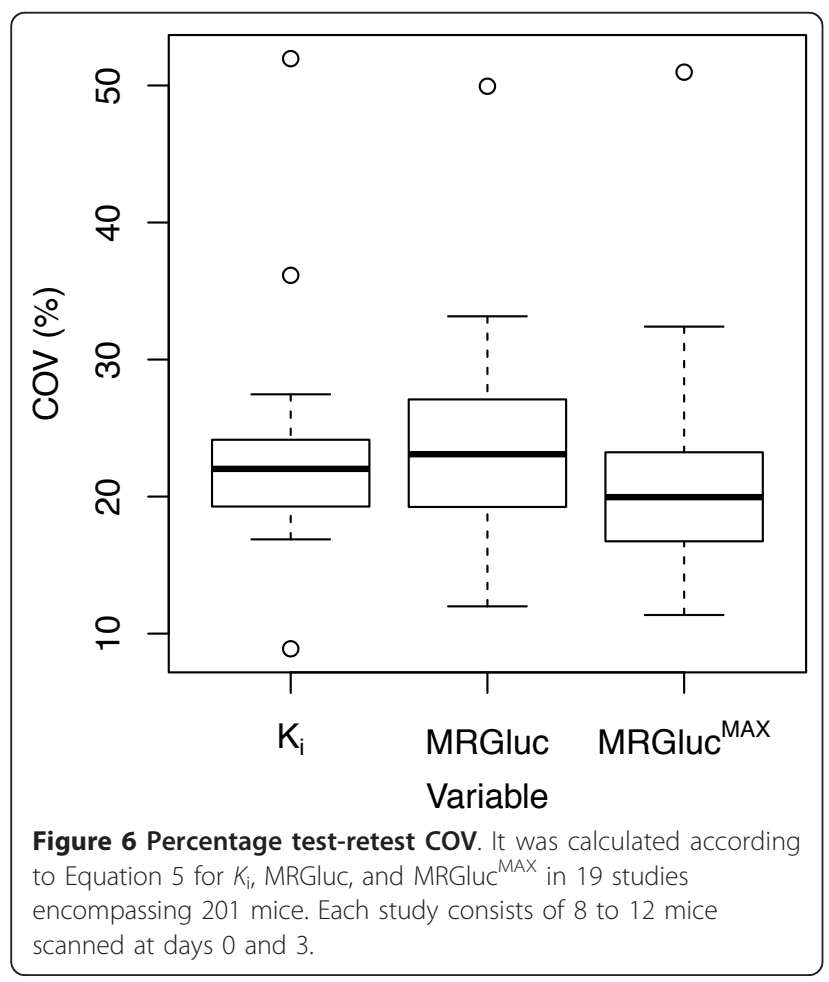

across a variety of tumor models and mouse strains. It is possible to calculate confidence intervals for the correlation coefficients; these reinforce our conclusions since only one of eleven models had a 95\% confidence interval that included zero (-0.01 to 0.42). These calculations and results are presented in Additional file 2 for the interested reader.

\section{Use and applicability of MRGluc}

Rigorous methods for estimating the MRGluc utilization were developed over 30 years ago and continue to be successfully applied $[7,12,26,30,31]$, not least in tumors $[2,4,17,32-36]$. However, capturing the rate of glucose uptake in the instant of the scan leads to MRGluc reflecting changes in blood glucose whether or not they are functionally significant to the tissue. For malignant tumors, which are highly glucose-addicted, glucose uptake capacity may well be a more important tissue characteristic to consider than the glucose uptake rate. MRGluc $^{\mathrm{MAX}}$ reduces glucose bias by emphasizing capacity rather than rate.

\section{Fundamental problem with nonlinear regression estimates of $K_{\mathrm{M}}$ and MRGluc ${ }^{\mathrm{MAX}}$}

It may be surprising to some readers that we do not employ a nonlinear regression model to simultaneously estimate MRGluc $^{\mathrm{MAX}}$ and $K_{\mathrm{M}}$ from measurements of $K_{\mathrm{i}}$ and [glucose]. Although considerable care must be taken, this approach is known to work [37-39] for enzymatic data collected in vitro with minimal statistical noise in the measurements. However, it proved to be impossible with our data from living subjects: the objective function was difficult to optimize and subject to very large estimation errors. Mathematically, this is due to maximum likelihood estimates of $K_{\mathrm{M}}$ and MRGlucMAX being highly linearly codependent, and it requires a wide range of glucose values to confidently distinguish the effects of changing $K_{\mathrm{M}}$ and changing MRGluc ${ }^{\mathrm{MAX}}$, at least when faced with relatively noisy real-world $K_{\mathrm{i}}$ measurements. This argument is presented in Appendix 1 for the interested reader along with simulations.

\section{Use of a fixed value of $K_{\mathrm{M}}$}

The use of an apparent $K_{\mathrm{M}}$ value derived in separate experiments and used within a physiologically reasonable range has the mathematical advantage that it reduces the number of parameters we need to estimate from the scan data and thus avoids the use of underpowered determinations made on a case-by-case basis. We used a large sample of studies to determine the $K_{\mathrm{M}}$ at which there was, on average, no net correlation between MRGluc ${ }^{\mathrm{MAX}}$ and blood glucose levels $\left(K_{\mathrm{M}}=\right.$ $130 \mathrm{mg} / \mathrm{dL}$ ). We regard this as an upper limit; the lower limit might be set by studies on isolated cells where the 
measurement can be made with full knowledge of the extracellular glucose concentration $\left(K_{\mathrm{M}}=40 \mathrm{mg} / \mathrm{dL}\right.$, [9]).

A biological advantage is that we are better able to fix the $K_{\mathrm{M}}$ as a constant property of a certain tissue or tumor type under given conditions which are largely dictated by the discrete nature of the molecular determinants, such as the isotype of the glucose transporter, GLUT-1 versus GLUT-3, for example.

\section{The importance of tissue glucose and blood glucose}

Of particular importance should be how the (mechanistically relevant) tissue glucose relates to the (conveniently measured) blood glucose levels [40]. Ideally, we should know the interstitial glucose concentration in the tumor microenvironment, and while the relationship between blood and tissue glucose is of intense interest and active study, it is still not trivial to measure [41-43]. For normal tissues, interstitial glucose may be modeled, but in tumors with all their heterogeneity and variability, this is likely to remain challenging, and this will likely continue to present a significant source of variability in data that depend on tissue glucose but measure blood glucose.

In some tumors, the glucose utilization is so great and the perfusion so poor that the true tissue glucose may be close to zero [44], leaving tissue glucose transport far from being saturated yet also decoupled from blood glucose levels. We have seen from our data is possible, but not typical. More common are cases where the FDG uptake rate does correlate with blood glucose levels, implying some degree of saturation and thus nonzero tissue glucose levels.

\section{Mathematical expectation of a correlation between $K_{\mathrm{i}} \times$ [glucose] and [glucose]}

Here, we report an empirical correlation between glucose as it is commonly measured (in the blood) and MRGluc as it is commonly defined and described in the literature $\left(K_{\mathrm{i}} \times\right.$ [glucose], based on blood glucose measurements). Although it is not widely remarked upon in the literature, this correlation appears to be almost inevitable, a natural consequence of the relationship between $K_{\mathrm{i}}$, MRGluc, and [glucose]. Given the widely described result that tissue FDG uptake rates $\left(K_{\mathrm{i}}\right)$ and FDG uptake levels (standardized uptake value [SUV]) are affected by [glucose] [2-6,45-48], truly incredible circumstances must prevail to have zero correlation between MRGlu and [glucose] under all circumstances. A more extensive mathematical analysis of this problem is presented for the interested reader in the Appendix 2.

\section{Applicability of $K_{\mathrm{M}}$ values across multiple tumor types}

Model-specific $K_{\mathrm{M}}$ values might be expected to have some benefit and were tested as an exploratory measure. They made it possible to bring the blood glucose correlation with MRGluc ${ }^{\mathrm{MAX}}$ close to zero for each tumor model independently. However, there was no additional improvement in the between-animal variability. Employing a global value of $130 \mathrm{mg} / \mathrm{dL}$ seemed adequate for these exploratory studies given that the benefits of using MRGluc ${ }^{\mathrm{MAX}}$ are not critically dependent on using a precise value of $K_{\mathrm{M}}$.

\section{Alternative linear regression method for estimation of MRGluc $^{\text {MAX }}$ with a fixed $K_{M}$}

Having adopted the use of a fixed value for $K_{\mathrm{M}}$, we note that a least-squares linear regression method to compute MRGluc $^{\mathrm{MAX}}$ is readily apparent from Equation 3 by plotting $K_{\mathrm{i}}$ as a function of $\left(1 /\left(K_{\mathrm{M}}+\right.\right.$ [glucose] $)$, giving a straight line with MRGluc ${ }^{\mathrm{MAX}}$ as the slope when the regression line is forced through the origin. Estimation of the group mean by linear regression may perform better than averaging individual values (c.f., Section II.5 in the book by Christensen [49]). However, when we tested this alternative calculation, we found that it made no appreciable difference to the results. We note that linear regression should offer the greatest benefit where the data contain a wide spread of glucose concentrations; as we noted above, this is not the case for our living-subject data with its relatively narrow range of physiological blood glucose values and relatively high noise level.

\section{Variability and statistical power of MRGluc ${ }^{\mathrm{MAX}}$ compared to MRGluc}

As noted, a $40 \%$ increase in sample size would be required to achieve a $15.5 \%$ reduction in COV. However, translating a reduction in COV to improve statistical power requires additional assumptions, e.g., regarding the potential treatment effect [50]. To make a preliminary estimate, we assume that the relative treatment effect is the same for MRGluc ${ }^{\mathrm{MAX}}$ and MRGluc when expressed as a percentage change from baseline (a conservative assumption since MRGluc ${ }^{\mathrm{MAX}}$ is an asymptote). In this case, the reduction in the required sample size while maintaining the same error rates (i.e., the same statistical power) is $28.6 \%$ (equal to $100 \times(1-(1-$ $\left.0.155)^{2}\right)$ ) (see Equation 2 in van Belle and Martin [50]). The actual sample size savings achieved in practice are likely to be smaller than this because assumptions will not hold exactly. In particular, glucose uptake is only approximated by Michaelis-Menten kinetics; $K_{\mathrm{M}}$ is not known exactly; and the error distribution may be neither Gaussian nor perfectly homoscedastic.

No doubt there are many sources of physiological noise contributing to the total observed variability in $K_{\mathrm{i}}$ [51], and blood glucose may be only a small part of that. Nevertheless, a $15.5 \%$ reduction in between-animal $\mathrm{COV}$ is not trivial and could well become important over the course of many studies or in marginal cases. 
Also, this improvement should not be considered in isolation, but seen as one step in the evolution of PET methodology over the years.

\section{Glucose normalization and bias}

Although biologically appealing, mixed results have come from previous studies of linear glucose normalizations applied to FDG-PET data [3-5]. Multiplying $K_{\mathrm{i}}$ by blood glucose (or normalized glucose, i.e., [glucose]/100 $\mathrm{mg} / \mathrm{dL}$ ) did not eliminate bias in our data. Some have found that this normalization actually increased variability and was unhelpful $[8,52,53]$, possibly because of the noise introduced by the glucose assay. However, glucose bias was significantly reduced with the nonlinear MRGluc ${ }^{\text {MAX }}$ function, while simultaneously achieving reductions in between-animal and test-retest COVs compared to both $K_{\mathrm{i}}$ and MRGluc. This is very encouraging and warrants further investigation.

\section{Other nonlinear glucose corrections}

Given the significant biological noise that remains in FDG-PET data even after various corrections are applied, other line equations that approximate the Michaelis-Menten equation should fit the data and give broadly similar bias reductions and improvements in glucose-derived variability. For example, Wong et al. have demonstrated that using a square-root function of the glucose concentration allowed their clinical SUV data to better classify indolent and aggressive lymphomas [54]. They also suggested, referring to Langen et al. [2], that this correction would not be necessary with dynamic scans quantified with MRGluc.

\section{Applicability to SUV data}

Our preliminary observations confirm that tumor SUV values correlate highly with our $K_{\mathrm{i}}$ data, showing a negative correlation with blood glucose across hundreds of mice and dozens of studies. However, our SUV values were derived from time-activity curves at no more than 30 min after injection of FDG, and with only 5 min of acquisition time, making them statistically noisy compared to purposeful SUV data. We expect that partial saturation correction will have similar benefits with SUV data, but more appropriate experimental data will be required before this can be properly explored. However, applying the square root of glucose SUV correction of Wong et al. [54] to our tumor data did reduce glucose bias and variability compared to MRGluc, almost as much as MRGluc ${ }^{\mathrm{MAX}}$. The converse should also be true, suggesting that multiplying SUV by $\left(K_{\mathrm{M}}+\right.$ [glucose]) would be effective in the clinical lymphoma setting, while the mechanistic foundation of this correction may make it possible to rationally optimize $K_{\mathrm{M}}$ in different tissues or tumor types.

\section{Outliers and blood glucose changes during the scan}

Individual outliers often exhibited large differences between their pre-scan and post-scan blood glucose levels. We tested some exclusion criteria which censored out data from scans where there had been a $75 \%$ or greater change in blood glucose level during the course of the scan. This helped reduce the between-animal variability in some studies. However, a more attractive alternative may be to track and account for the changes in blood glucose occurring during a scan as proposed by Dunn et al. [55]. It would be interesting to evaluate a combination of partial saturation correction and the method of Dunn et al. [55] to better account for both between-scan and intra-scan blood glucose changes.

\section{Conclusions}

Measured in a very large sample of 1,192 nonclinical dynamic FDG-PET scans, it was clear that the rate of tumor glucose uptake estimated by MRGluc was, in most studies, positively correlated with blood glucose levels. This gave an unwanted bias and additional variability in our estimates of tumor glucose uptake rates.

By assuming a Michaelis-Menten relationship, the simple use of $K_{\mathrm{M}}+$ [glucose] in place of [glucose] as the glucose correction factor had several benefits: the hypothetical glucose-saturated MRGluc ${ }^{\text {MAX }}$ was less correlated with blood glucose, had lower between-animal variability, and had lower test-retest variability compared to MRGluc.

\section{Future directions}

This reduced bias and reduced variability may translate into a significant reduction in sample size (up to 28\%) for nonclinical treatment studies. Further performance comparisons of MRGluc and MRGluc ${ }^{\text {MAX }}$ applied to detect confirmed treatment responses in our nonclinical tumor models have been completed and will be described separately.

It will be very interesting, and straightforward, to see if these findings can be translated to studies of clinical trial data where saturation-corrected SUV data could be calculated by multiplying SUV by $(100 \mathrm{mg} / \mathrm{dL}+$ [glucose]), rather than the more commonly reported glucose-normalized SUV employing ([glucose]/100 mg/dL). In the clinical trial setting, even modest reductions in variability can translate to tangible savings in money, time, and patient enrollment.

\section{Appendix 1}

On the problem of linearly dependent ML estimates of $K_{\mathrm{m}}$ and $V_{\max }$ from noisy Michaelis-Menten observations If an adequate probabilistic framework can be specified for a sample data set, maximum likelihood [ML] typically provides an efficient approach for parameter 
estimation. Equivalently, for data which are conditionally Gaussian-distributed, one may also use nonlinear least squares. However, due to the functional form of the Michaelis-Menten [MM] relationship, $K_{\mathrm{m}}$ and $V_{\max }$ are not uniquely estimable (from each other) from noisy MM observations. This problem is further exacerbated when the MM process is observed in a narrow glucose range (as is the case in our work). The problem can be understood by studying the information matrix for $K_{\mathrm{m}}$ and $V_{\max }$, but we use a first-order expansion of the ML score function to heuristically verify that the ML parameter estimates of $K_{\mathrm{m}}$ and $V_{\max }$ are strongly co-linear.

Let the true values of $K_{\mathrm{m}}$ and $V_{\max }$ be given by $K_{\mathrm{o}}$ and $V_{\mathrm{o}}, \quad$ respectively. For $j=1, \ldots, n$, let $K_{\mathrm{i}}^{j}=V_{\mathrm{o}} /\left(K_{\mathrm{o}}+[g l c]^{j}\right)+\varepsilon^{j}$ be the $j$ :th observed rate constant where $\varepsilon^{j}$ is independently sampled from a zeromean Gaussian distribution with standard deviation $\sigma$.

Let $S\left(V_{\max }, K_{\mathrm{m}}, \sigma\right)=\sigma^{-2} \sum_{j}\left(K_{\mathrm{i}}^{j}-V_{\max } /\left(K_{\mathrm{m}}+[g l c]^{j}\right)\right)^{2}$ be the ML score function to be minimized. The ML estimates are given by

$$
\left\{\hat{V}_{\max }, \hat{K}_{\mathrm{m}}, \hat{\sigma}\right\}=\min _{V_{\max }, K_{\mathrm{m}}, \sigma} S\left(V_{\max }, K_{\mathrm{m}}, \sigma\right) .
$$

We note that estimation of $\sigma$ does not affect estimates of $V_{\max }$ and $K_{\mathrm{m}}$, and its consideration is henceforth eschewed.

For reasonably large sample sizes, at convergence, the ML estimates $\hat{V}_{\text {max }}, \hat{K}_{\text {m }}$ satisfy

$$
\begin{aligned}
S\left(\hat{V}_{\max }, \hat{K}_{\mathrm{m}}\right) \approx \sum_{j} & \left(\left(\varepsilon^{j}\right)^{2}-2 \varepsilon^{j} /\left(K_{\mathrm{o}}+\left[g l c c^{j}\right)\left[\left(\hat{V}_{\max }-V_{\mathrm{o}}\right)-V_{\mathrm{o}} /\left(K_{\mathrm{o}}+[g l c]^{j}\right)\left(\hat{K}_{\mathrm{m}}-K_{\mathrm{o}}\right)\right]\right.\right. \\
& +1 /\left(K_{\mathrm{o}}+[g l c]^{j}\right)^{2}\left[\left(\hat{V}_{\max }-V_{\mathrm{o}}\right)-V_{\mathrm{o}} /\left(K_{\mathrm{o}}+[g l c]^{j}\right)\left(\hat{K}_{\mathrm{m}}-K_{\mathrm{o}}\right)\right]^{2} .
\end{aligned}
$$

Taking expectations over the noise process, $\varepsilon^{j}$ yields that on the average, the score is minimized approximately when $\left(\hat{V}_{\max }-V_{\mathrm{o}}\right)=V_{\mathrm{o}} /\left(K_{\mathrm{o}}+[m \cdot g l c]\right)\left(\hat{K}_{\mathrm{m}}-K_{\mathrm{o}}\right)$, where $[m . g l c]$ represents the mean glucose measurement. The result holds when the glucose measurements have low spread but can be shown to hold approximately even as the spread around $[\mathrm{m} . \mathrm{glc}]$ increases. Thus, $\hat{V}_{\max }$ is linear in $\hat{K}_{\mathrm{m}}$ with a slope equal to $V_{\mathrm{o}} /\left(K_{\mathrm{o}}\right.$ $+[m . g l c])$.

To illustrate, we ran 400 simulations with the following parameters: $V_{\max }=V_{\mathrm{o}}=40, K_{\mathrm{m}}=K_{\mathrm{o}}=100, \sigma=$ .025, where glucose was randomly sampled from a Gaussian distribution with the mean $[\mathrm{m} \cdot g l c]=100$ and standard deviation of 20. Each such simulation used a total of $n=20$ observations. These parameter settings were chosen to simulate data which closely mimics the previously presented data. The R function $n l s()$ was used for ML estimation. Figure 7 shows pairs of estimates of $V_{\max }$ (i.e., $\hat{V}_{\max }$ on the $y$-axis $)$ and $K_{\mathrm{m}}\left(\hat{K}_{\mathrm{m}}\right.$ on the $x$ axis) from these 400 simulations. As can be seen, the

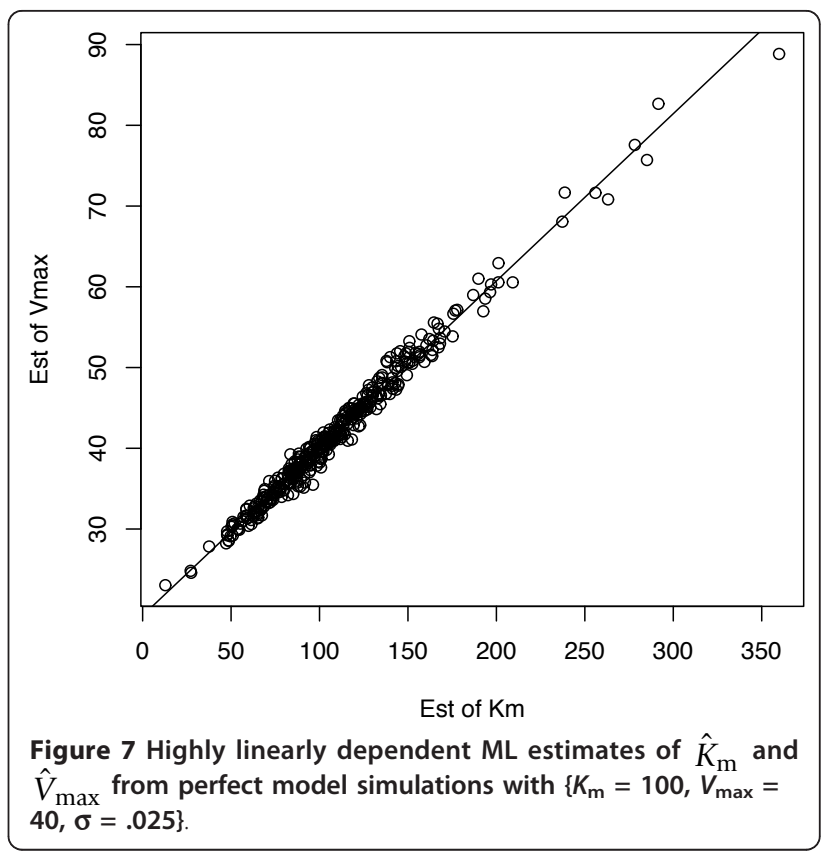

ML estimates are highly linearly dependent and have a slope of 0.2072 , very near to that derived by $V_{\mathrm{o}} /\left(K_{\mathrm{o}}+[\mathrm{m}\right.$. $g l c])=.20$. Further, the sample correlation in this plot is .995 , indicating that estimates are not uniquely identifiable from the data.

Although slightly improved, simulations verify that the above problem persists even as the spread in $[g l c]$ is greatly increased. Figure 8 sheds some intuition on the overall problem. Along with the black MM curve used

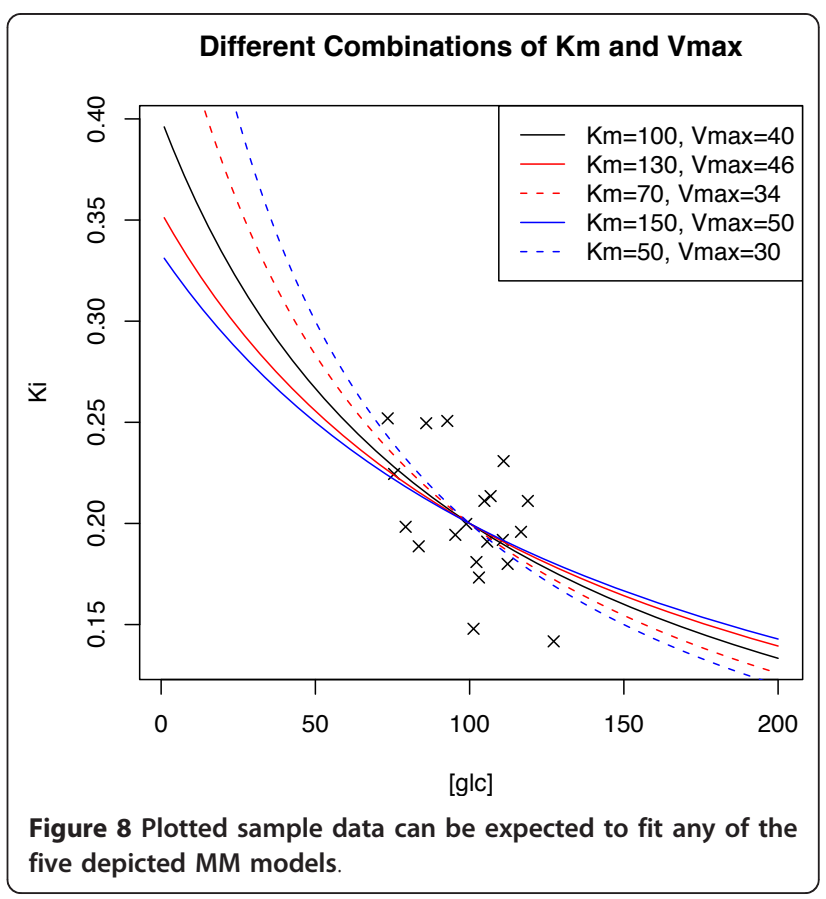


to generate 20 sample data points (denoted by black ' $x$ ') are the MM curves for four other parameter settings (see figure legend for parameter values). As is evident, the sample data cannot be expected to 'choose' efficiently among the depicted candidate models as increases in $K_{\mathrm{m}}$ are 'traded' for increases (and vice versa) in $V_{\max }$ at the rate of .2. This estimation issue remains even as the sample size is increased albeit with decreasing overall sample variability at a rate of $1 /$ sqrt (n).

\section{Appendix 2}

\section{On the correlation between MRgluc and [g/c]}

As noted, several authors have described studies where the observed rates $K_{\mathrm{i}}$ were negatively correlated with the glucose measurements $[g l c]$. We show that when such a negative correlation exists, the correlation between $M R g l u c$, defined as the product between $K_{\mathrm{i}}$ and $[g l c]$, and $[g l c]$ cannot uniformly equal zero. Indeed, lack of correlation occurs but in a few special cases.

Since $K_{\mathrm{i}}>0$ and $[g l c]>0$ are negatively correlated, there exist constants $\alpha>0$ and $\beta<0$ such that the form $K_{\mathrm{i}} \approx \alpha+\beta[g l c]+\varepsilon$ describes the correlation between $K_{\mathrm{i}}$ and $[g l c]$. Here, $\varepsilon$ is a zero-mean error process independent of $[g l c]$ with variance $\sigma_{\varepsilon}^{2}$. Then, with $\mu_{g}^{k}$ denoting the $k$ :th raw moment of $[g l c]$ and with $\sigma_{g}^{2}$ its variance, straightforward algebra shows that the covariance between $M R g l u c$ and $[g l c]$ equals

$$
\operatorname{cov}(\text { MRgluc, }[g l c])=\alpha \sigma_{g}^{2}+\beta\left(\mu_{g}^{3}-\mu_{g}^{2} \mu_{g}\right) .
$$

Thus, for $\operatorname{cov}($ MRgluc, $[g l c])=0$, we must have $\alpha \sigma_{g}^{2}=-\beta\left(\mu_{g}^{3}-\mu_{g}^{2} \mu_{g}\right)$ or $\alpha / \beta=\left(\mu_{g}^{2} \mu_{g}-\mu_{g}^{3}\right) / \sigma_{g}^{2}$. This equality can clearly not hold uniformly across a broad range of realistic parameter choices of $\left\{\alpha, \beta, \mu_{g}, \mu_{g}^{2}, \mu_{g}^{3}\right\}$.

We illustrate the result by simulations. Our setting assumes that $K_{\mathrm{i}}$ follows the MM form with constants $K_{\mathrm{m}}$ and $V_{\max }$ and that the observed rate is corrupted by noise. That is, $K_{\mathrm{i}}=V_{\max } /\left(K_{\mathrm{m}}+[g l c]\right)+\varepsilon$, where $\varepsilon$ is the random Gaussian with zero-mean and standard deviation $\sigma$. As is common, we further assume that the rate constant is observed (sampled) in a glucose range between 60 and 140 . We note that when $K_{\mathrm{i}}$ is observed in a limited range around some glucose midpoint $[\mathrm{m}$. $g l c], K_{\mathrm{i}} \approx\left(V_{\max } /\left(K_{\mathrm{m}}+[m \cdot g l c]\right)+\left([m \cdot g l c] V_{\max }\right) /\left(K_{\mathrm{m}}+[m\right.\right.$. $\left.g l c])^{2}\right)-V_{\max } /\left(K_{\mathrm{m}}+[m . g l c]\right)^{2}[g l c]+\varepsilon$, i.e., $K_{\mathrm{i}}$ is approximately linear in $[g l c]$.

The left panel in Figure 9 shows 400 simulated observations drawn from a MM model with $V_{\max }=40, K_{\mathrm{m}}=$ $100, \sigma=.025$, where glucose was randomly sampled from a Gaussian distribution with a mean of 100 and a standard deviation of 15. As can be seen, in the sampled range, $K_{\mathrm{i}}$ is approximately linear in $[g l c]$. The right panel shows a scatter plot of $[g l c]$ vs. MRgluc. Consistent with our derivations, the sample correlations in the two plots are -.48 and .53 , respectively. For the chosen parameter choices and glucose distribution, based on the above arguments, the sample correlation between $[g l c]$ and MRgluc should be near to its theoretically predicted value of .51. (For this data, the sample correlation between $[g l c]$ and $M R g l u c^{M A X}=K_{\mathrm{i}}\left(K_{\mathrm{m}}+[g l c]\right)$ is .01.)

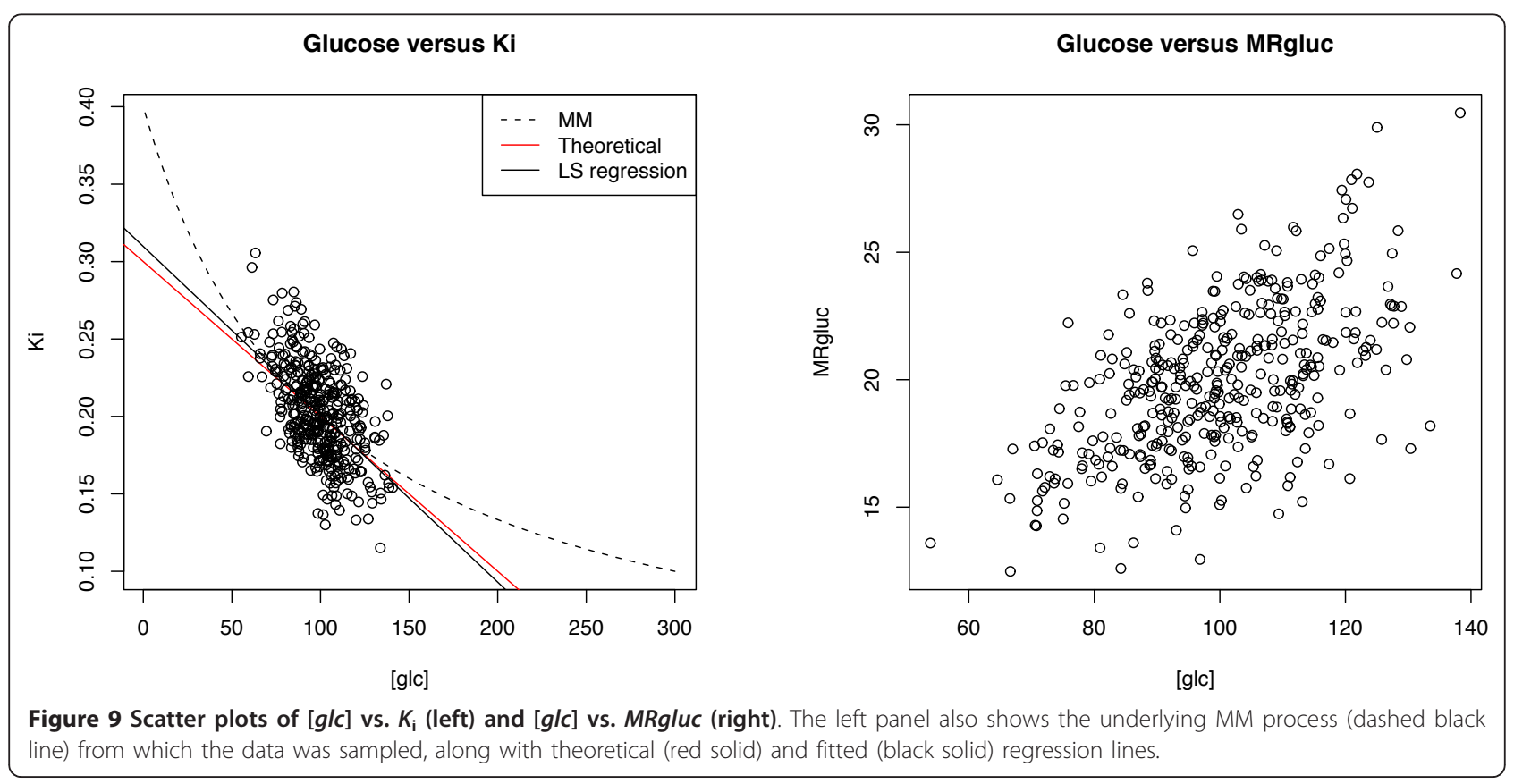




\section{Additional material}

Additional file 1: ROI data and corresponding Patlak plots from FDG-PET scans in each of the 11 tumor models $A$ to $K$ discussed in the text (see Table 1). In each plot, the data from one cohort $(n=14$ to 36) of essentially identical mice are superimposed. Left, in red: the liver-derived input function; center, in blue: the tumor; right, in gray: the Patlak plot.

Additional file 2: Confidence intervals for correlations between PET metrics and blood glucose. To obtain the $95 \%$ confidence limits for Pearson's correlation coefficient $(r)$, the Fisher transformation was applied to the sample correlation coefficients.

\section{Acknowledgements}

The authors gratefully acknowledge the contributions of Annie Ogasawara, Alex Vanderbilt, Jeff Tinianow, Herman Gill, Leanne McFarland, and Karissa Peth who helped execute the imaging studies analyzed here.

\section{Author details}

'Department of Biomedical Imaging, Genentech, Inc., South San Francisco, CA, 94080, USA ${ }^{2}$ Department of Pharmacokinetics and Pharmacodynamics, Genentech, Inc., South San Francisco, CA, 94080, USA ${ }^{3}$ Department of Biostatistics, Genentech, Inc., South San Francisco, CA, 94080, USA

\section{Authors' contributions}

S-PW designed the studies and wrote the manuscript, JEF-M programmed the data analyses and prepared the figures, REP guided the discussion, and TB guided the data analysis and statistics. All authors read and approved the final manuscript.

\section{Competing interests}

The authors declare that they have no competing interests.

Received: 21 September 2011 Accepted: 1 February 2012

Published: 1 February 2012

\section{References}

1. Crouthamel MC, Kahana JA, Korenchuk S, Zhang SY, Sundaresan G, Eberwein DJ, Brown KK, Kumar R: Mechanism and management of AKT inhibitor-induced hyperglycemia. Clin Cancer Res: Official J Am Assoc Cancer Res 2009, 15:217-225.

2. Langen KJ, Braun U, Rota Kops E, Herzog H, Kuwert T, Nebeling B, Feinendegen LE: The influence of plasma glucose levels on fluorine-18fluorodeoxyglucose uptake in bronchial carcinomas. J Nucl Med 1993, 34:355-359.

3. Lindholm P, Minn H, Leskinen-Kallio S, Bergman J, Ruotsalainen U, Joensuu $\mathrm{H}$ : Influence of the blood glucose concentration on FDG uptake in cancer-a PET study. J Nucl Med 1993, 34:1-6.

4. Huang SC: Anatomy of SUV. Standardized uptake value. Nucl Med Biol 2000, 27:643-646.

5. Keyes JW: SUV: standard uptake or silly useless value? J Nucl Med 1995, 36:1836-1839.

6. Diederichs C, Staib L, Glatting G, Beger H, Reske S: FDG PET: elevated plasma glucose reduces both uptake and detection rate of pancreatic malignancies. J Nucl Med 1998, 39:1030-1033.

7. Sokoloff $L$, Reivich M, Kennedy C, Des Rosiers MH, Patlak CS, Pettigrew KD, Sakurada O, Shinohara M: The [14C]deoxyglucose method for the measurement of local cerebral glucose utilization: theory, procedure, and normal values in the conscious and anesthetized albino rat. $J$ Neurochem 1977, 28:897-916.

8. Hallett WA, Marsden PK, Cronin BF, O'Doherty MJ: Effect of corrections for blood glucose and body size on [18F]FDG PET standardised uptake values in lung cancer. Eur J Nucl Med 2001, 28:919-922.

9. Kasahara M, Hinkle PC: Reconstitution and purification of the d-glucose transporter from human erythrocytes. J Biol Chem 1977, 252:7384-7390.

10. Rivenzon-Segal D, Rushkin E, Polak-Charcon S, Degani H: Glucose transporters and transport kinetics in retinoic acid-differentiated T47D human breast cancer cells. Am J Physiol Endocrinol Metab 2000, 279: E508-519.

11. Rodriguez-Enriquez S, Marin-Hernandez A, Gallardo-Perez JC, MorenoSanchez R: Kinetics of transport and phosphorylation of glucose in cancer cells. J Cell Physiol 2009, 221:552-559.

12. Van Zijl PC, Davis D, Eleff SM, Moonen CT, Parker RJ, Strong JM: Determination of cerebral glucose transport and metabolic kinetics by dynamic MR spectroscopy. Am J Physiol 1997, 273:E1216-1227.

13. Cornford EM, Young D, Paxton JW, Hyman S, Farrell CL, Elliott RB: Bloodbrain glucose transfer in the mouse. Neurochem Res 1993, 18:591-597.

14. Lammertsma AA, Hoekstra CJ, Giaccone G, Hoekstra OS: How should we analyse FDG PET studies for monitoring tumour response? Eur J NuCl Med Mol Imaging 2006, 33:16-21.

15. Patlak CS, Blasberg RG, Fenstermacher JD: Graphical evaluation of bloodto-brain transfer constants from multiple-time uptake data. J Cerebral Blood Flow Metab 1983, 3:1-7.

16. Patlak CS, Blasberg RG: Graphical evaluation of blood-to-brain transfer constants from multiple-time uptake data. Generalizations. J Cereb Blood Flow Metab 1985, 5:584-590.

17. Cheebsumon P, Velasquez LM, Hoekstra CJ, Hayes W, Kloet RW, Hoetjes NJ, Smit EF, Hoekstra OS, Lammertsma AA, Boellaard R: Measuring response to therapy using FDG PET: semi-quantitative and full kinetic analysis. Eur $J$ Nucl Med Mol Imaging 2011, 38:832-842.

18. Flores JE, Mcfarland LM, Vanderbilt A, Ogasawara AK, Williams S-P: The effects of anesthetic agent and carrier gas on blood glucose and tissue uptake in mice undergoing dynamic FDG-PET imaging: sevoflurane and isoflurane compared in air and in oxygen. Mol Imaging Biol 2008, 10:192-200.

19. Li Q, Leahy R: Statistical modeling and reconstruction of randoms precorrected PET data. IEEE Trans Med Imaging 2006, 25:1565-1572.

20. Bao Q, Newport D, Chen M, Stout DB, Chatziioannou AF: Performance evaluation of the inveon dedicated PET preclinical tomograph based on the NEMA NU-4 standards. J Nucl Med 2009, 50:401-408.

21. Green L, Gambhir S, Srinivasan A, Banerjee P, Hoh C, Cherry S, Sharfstein S, Barrio J, Herschman H, Phelps M: Noninvasive methods for quantitating blood time-activity curves from mouse PET images obtained with fluorine-18-fluorodeoxyglucose. J Nucl Med 1998, 39:729-734.

22. Meyer PT, Circiumaru V, Cardi CA, Thomas DH, Bal H, Acton PD: Simplified quantification of small animal [18F]FDG PET studies using a standard arterial input function. Eur I Nucl Med Mol Imaging 2006, 33:948-954.

23. R Development Core Team: R: A Language and Environment for Statistical Computing Vienna: R Foundation for Statistical Computing; 2011.

24. Logan J: Graphical analysis of PET data applied to reversible and irreversible tracers. Nucl Med Biol 2000, 27:661-670.

25. Doot RK, Dunnwald LK, Schubert EK, Muzi M, Peterson LM, Kinahan PE, Kurland BF, Mankoff DA: Dynamic and static approaches to quantifying 18F-FDG uptake for measuring cancer response to therapy, including the effect of granulocyte CSF. J Nucl Med 2007, 48:920-925.

26. Krohn KA, Mankoff DA, Muzi M, Link JM, Spence AM: True tracers: comparing FDG with glucose and FLT with thymidine. Nucl Med Biol 2005, 32:663-671.

27. Cornish-Bowden A: Fundamentals of Enzyme Kinetics London: Portland Press; 2004

28. Michaelis L, Menten ML: Die kinetik der invertinwirkung. Biochemische Zeitschrift 1913, 49:333-369.

29. Weber WA, Schwaiger M, Avril N: Quantitative assessment of tumor metabolism using FDG-PET imaging. NuCl Med Biol 2000, 27:683-687.

30. Phelps ME, Huang SC, Hoffman EJ, Selin C, Sokoloff L, Kuhl DE: Tomographic measurement of local cerebral glucose metabolic rate in humans with (F-18)2-fluoro-2-deoxy-d-glucose: validation of method. Ann Neurol 1979, 6:371-388.

31. Phelps ME, Schelbert HR, Hoffman EJ, Huang SC, Kuhl DE: Positron tomography of the heart. Prog Nucl Med 1980, 6:183-209.

32. de Geus-Oei L-F, Visser EP, Krabbe PFM, van Hoorn BA, Koenders EB, Willemsen AT, Pruim J, Corstens FHM, Oyen WJG: Comparison of imagederived and arterial input functions for estimating the rate of glucose metabolism in therapy-monitoring 18F-FDG PET studies. J Nucl Med 2006, 47:945-949.

33. Eary JF, Conrad EU, Bruckner JD, Folpe A, Hunt KJ, Mankoff DA, Howlett AT: Quantitative [F-18]fluorodeoxyglucose positron emission tomography in pretreatment and grading of sarcoma. Clin Cancer Res 1998, 4:1215-1220. 
34. Hawkins RA, Choi Y, Huang SC, Messa C, Hoh CK, Phelps ME: Quantitating tumor glucose metabolism with FDG and PET. J Nucl Med 1992, 33:339-344.

35. Hunter GJ, Hamberg LM, Alpert NM, Choi NC, Fischman AJ: Simplified measurement of deoxyglucose utilization rate. J Nucl Med 1996, 37:950-955.

36. Vriens D, De Geus-Oei LF, Van Laarhoven HWM, Van Der Heijden HFM, Krabbe PFM, Visser EP, Oyen WJG: Comparison of two region of interest definition methods for metabolic response evaluation with [18F]FDGPET. Q J Nucl Med Mol Imaging 2010, 54:677-688.

37. Dette H, Biedermann S: Robust and efficient designs for the MichaelisMenten model. J Am Stat Assoc 2003, 98:679-686.

38. Raaijmakers JGW: Statistical analysis of the Michaelis-Menten equation. Biometrics 1987, 43:793-803.

39. Ruppert D, Cressie N, Carroll RJ: A transformation weighting model for estimating Michaelis-Menten parameters. Biometrics 1989, 45:637-656.

40. Burrows RC, Freeman SD, Charlop AW, Wiseman RW, Adamsen TC Krohn KA, Spence AM: [18F]-2-fluoro-2-deoxyglucose transport kinetics as a function of extracellular glucose concentration in malignant glioma, fibroblast and macrophage cells in vitro. Nucl Med Biol 2004, 31:1-9.

41. Aussedat B, Dupire-Angel M, Gifford R, Klein JC, Wilson GS, Reach G: Interstitial glucose concentration and glycemia: implications for continuous subcutaneous glucose monitoring. Am J Physiol Endocrinol Metab 2000, 278:E716-728.

42. Thennadil SN, Rennert JL, Wenzel BJ, Hazen KH, Ruchti TL, Block MB: Comparison of glucose concentration in interstitial fluid, and capillary and venous blood during rapid changes in blood glucose levels. Diabetes Technol Ther 2002, 3:357-365.

43. Cengiz $\mathrm{E}$, Tamborlane $\mathrm{W}: \mathrm{A}$ tale of two compartments: interstitial versus blood glucose monitoring. Diabetes Technol Ther 2009, 11:S11-16.

44. Gullino PM, Clark SH, Grantham FH: The interstitial fluid of solid tumors. Cancer Res 1964, 24:780-794.

45. Wahl R, Henry C, Ethier S: Serum glucose: effects on tumor and normal tissue accumulation of 2-[F-18]-fluoro-2-deoxy-d-glucose in rodents with mammary carcinoma. Radiology 1992, 183:643-647.

46. Torizuka T, Fisher S, Wahl R: Insulin-induced hypoglycemia decreases uptake of 2-[F-18]fluoro-2-deoxy-d-glucose into experimental mammary carcinoma. Radiology 1997, 203:169-172.

47. Zhuang HM, Cortes-Blanco A, Pourdehnad M, Adam LE, Yamamoto AJ Martinez-Lazaro R, Lee JH, Loman JC, Rossman MD, Alavi A: Do high glucose levels have differential effect on FDG uptake in inflammatory and malignant disorders? Nucl Med Commun 2001, 22:1123-1128.

48. Gorenberg M, Hallett WA, O'Doherty MJ: Does diabetes affect [(18)F]FDG standardised uptake values in lung cancer? Eur I Nucl Med Mol Imaging 2002, 29:1324-1327.

49. Christensen R: Plane Answers to Complex Questions: The Theory of Linear Models New York: Springer; 1987.

50. van Belle G, Martin DC: Sample size as a function of coefficient of variation and ratio of means. Am Statistician 1993, 47:165-167.

51. Weber WA: Quantitative analysis of PET studies. Radiother Oncol 2010, 96:308-310.

52. Dandekar M, Tseng J, Gambhir S: Reproducibility of 18F-FDG microPET studies in mouse tumor xenografts. J Nucl Med 2007, 48:602-607.

53. Weber WA, Ziegler SI, Thodtmann R, Hanauske AR, Schwaiger M: Reproducibility of metabolic measurements in malignant tumors using FDG PET. J NuCl Med 1999, 40:1771-1777.

54. Wong CY, Thie J, Parling-Lynch KJ, Zakalik D, Margolis JH, Gaskill M, Hill J, Qing F, Fink-Bennett D, Nagle C: Glucose-normalized standardized uptake value from (18)F-FDG PET in classifying lymphomas. J Nucl Med: Official Publ, Soc Nucl Med 2005, 46:1659-1663.

55. Dunn JT, Anthony K, Amiel SA, Marsden PK: Correction for the effect of rising plasma glucose levels on quantification of MR(glc) with FDG-PET. $J$ Cereb Blood Flow Metab 2009, 29:1059-1067.

doi:10.1186/2191-219X-2-6

Cite this article as: Williams et al:: Quantitation of glucose uptake in tumors by dynamic FDG-PET has less glucose bias and lower variability when adjusted for partial saturation of glucose transport. EJNMMI Research 2012 2:6.

\section{Submit your manuscript to a SpringerOpen ${ }^{\mathcal{O}}$ journal and benefit from:}

- Convenient online submission

- Rigorous peer review

- Immediate publication on acceptance

- Open access: articles freely available online

- High visibility within the field

- Retaining the copyright to your article

Submit your next manuscript at $\gg$ springeropen.com 